\title{
A within-subject assessment of the discriminative stimulus and reinforcing effects of self-administered cocaine in rhesus monkeys
}

\author{
Jennifer L. Martelle • Michael A. Nader
}

Received: 2 June 2008 / Accepted: 30 August 2008 / Published online: 21 September 2008

(C) Springer-Verlag 2008

\begin{abstract}
Rationale Drug discrimination (DD) and drug self-administration (SA) are frequently used preclinical assays. All preclinical studies with cocaine have examined the discriminative stimulus $\left(\mathrm{S}^{\mathrm{D}}\right)$ and reinforcing $\left(\mathrm{S}^{\mathrm{R}}\right)$ effects in separate groups of subjects.

Objective The objective of the study is to train drug-naïve rhesus macaques to discriminate self-administered cocaine from saline and to assess $\mathrm{S}^{\mathrm{D}}$ and $\mathrm{S}^{\mathrm{R}}$ effects using a withinsubjects design.

Materials and methods Adult male rhesus monkeys $(n=4)$ were trained to self-administer cocaine $(0.1 \mathrm{mg} / \mathrm{kg}$ per injection) under a progressive-ratio (PR) reinforcement schedule. Next, they were trained to discriminate selfadministered cocaine $(0.45$ or $0.56 \mathrm{mg} / \mathrm{kg})$ or saline under a fixed-ratio (FR) 50 schedule of food presentation. The final schedule combined DD and SA into a multiple [chained FR 50 SA (cocaine or saline), food-reinforced DD] and PR SA schedule.

Results Each subject acquired SA under a PR schedule with significant differences in breakpoint between saline and cocaine evident by session 5 . Self-administered cocaine was established as an $\mathrm{S}^{\mathrm{D}}$, such that $80 \%$ of responding before delivery of the first reinforcer and $90 \%$ of all responding occurred on the injection-appropriate lever. In
\end{abstract}

\footnotetext{
J. L. Martelle $\cdot$ M. A. Nader $(\bowtie)$

Department of Physiology and Pharmacology,

Wake Forest University School of Medicine,

546 NRC, Medical Center Boulevard,

Winston-Salem, NC 27157-1083, USA

e-mail: mnader@wfubmc.edu

M. A. Nader

Department of Radiology,

Wake Forest University School of Medicine,

Winston-Salem, NC 27157, USA
}

all monkeys, there was at least one cocaine dose that did not engender cocaine-appropriate responding during DD (i.e., $<20 \%$ cocaine-appropriate responding) yet functioned as a reinforcer during PR SA, suggesting that cocaine-like $\mathrm{S}^{\mathrm{D}}$ effects are not necessary for cocaine reinforcement. Conclusions This within-subject model may provide new information related to the behavioral mechanisms of action leading to the high abuse potential of cocaine; such information may lead to novel pharmacological treatment strategies for addiction.

Keywords Cocaine - Drug discrimination .

Drug self-administration · Within-subjects · Rhesus monkey

\section{Introduction}

Drug discrimination (DD) and drug self-administration (SA) in laboratory animals are well-established models of drug abuse used to study discriminative stimulus $\left(\mathrm{S}^{\mathrm{D}}\right)$ and reinforcing stimulus $\left(\mathrm{S}^{\mathrm{R}}\right)$ effects, respectively (Griffiths et al. 1980; Bigelow and Preston 1989; Johanson and Fischman 1989; Woolverton and Nader 1990; Fischman and Foltin 1991; Mello and Negus 1996; Dykstra et al. 1997; Colpaert 1999; Grant 1999; Lelas et al. 2000). Both DD and SA have been successfully tailored for human studies with findings similar to those of nonhuman studies (Fischman 1988; Kamien et al. 1993).

In laboratory animals, it has been suggested that the $\mathrm{S}^{\mathrm{D}}$ effects are analogous to human subjective effects (Schuster and Balster 1977; Holtzman 1985). Further, while there are known deviations to the rule (e.g., hallucinogens; Carroll 1990), there is general agreement between the ability of a test drug to produce $\mathrm{S}^{\mathrm{D}}$ effects similar to those of a known drug of abuse and the likelihood that the test drug will be 
self-administered. For example, GBR 12909, an indirectacting dopamine (DA) agonist, not only substituted for cocaine in DD experiments, but also had $\mathrm{S}^{\mathrm{R}}$ effects in SA studies (Kleven et al. 1990; Witkin et al. 1991; Terry et al. 1994; Roberts 1993). There is an assertion that the drug actions governing $\mathrm{S}^{\mathrm{D}}$ effects are concomitant with those involved in $\mathrm{S}^{\mathrm{R}}$ effects as measured in $\mathrm{SA}$ (Schuster et al. 1981; Brady and Lukas 1984; Griffiths et al. 1985; Holtzman 1990). However, studies in human cocaine and stimulant abusers have noted discrepancies that suggest that the relationship between the production of $\mathrm{S}^{\mathrm{D}}$ and $\mathrm{S}^{\mathrm{R}}$ effects is not as concrete as first assumed (Fischman 1989; Johanson and Uhlenhuth 1980, 1981, 1982). For example, when human subjects were allowed to choose between $d$ amphetamine and placebo, they initially preferred the drug and reported positive subjective effects (Johanson and Uhlenhuth 1980, 1981). However, subjects chose to stop taking the drug despite the fact that the subjective effects were unchanged (during forced "sampling" administration) from when the drug was initially preferred. In this example, $d$-amphetamine was discriminated from placebo by its positive effect on mood, yet it did not function as a reinforcer. Similar results have been obtained from a preclinical study investigating the $S^{R}$ and $S^{D}$ effects of benzodiazepines (Ator 2002). Additionally, consideration of the individual characteristics of each subject can affect the interpretation of group data regarding any relationship between subjective (i.e., $S^{D}$ ) and $S^{R}$ effects (see Evans and Griffiths 1992; de Wit et al. 1986a, b). Uhlenhuth et al. (1981) demonstrated that the baseline subjective state of subjects but not drug-induced subjective effects determined drug preference. In all subjects, $d$-amphetamine produced comparable positive subjective effects compared to placebo. However, subjects that were more anxious and depressed prior to drug ingestion showed a marked preference for $d$-amphetamine and administered the drug at every opportunity compared to other subjects who did not always chose to administer the drug. Thus, subjective effects of the drug did not predict reinforcing effects.

To date, these individual differences have not been investigated in preclinical models of cocaine abuse, and all preclinical studies with cocaine have examined the $S^{D}$ and $S^{R}$ effects in separate groups of subjects. Therefore, the primary goal of the present study was to combine two established behavioral models of drug abuse-food-reinforced DD and intravenous drug SA - to create a unique operant paradigm examining the $S^{D}$ and $S^{R}$ effects of cocaine in the same subjects. Using a within-subjects design to examine DD and SA may provide added insight into the relationship between the production of interoceptive effects and the ability of cocaine to serve as a reinforcer that would otherwise not be apparent when examined in separate groups of subjects. Based on the above studies of human stimulant users
(Fischman 1989; Johanson and Uhlenhuth 1980, 1981, 1982) showing that $S^{D}$ and $S^{R}$ effects were not always aligned, it was hypothesized that there would be cocaine doses in which $S^{\mathrm{D}}$ effects would not be predictive of $\mathrm{S}^{\mathrm{R}}$ effects.

There were several novel features to the experiment. First, monkeys discriminated self-administered cocaine or saline. This was accomplished using a chained schedule in which fixed-ratio (FR) responding in the first link resulted in an intravenous injection of cocaine or saline, leading to stimulus changes within the chamber and initiation of foodreinforced discrimination in the second link. The reinforcing effects of that self-administered stimulus were assessed using a progressive-ratio (PR) schedule of reinforcement. There were several reasons for using a PR schedule in the second component of the multiple schedule. We have extensive experience using this schedule to examine the reinforcing strength of cocaine and other drugs of abuse (e.g., Lile et al. 2000, 2003; Czoty et al. 2006), so direct comparisons with published data are possible. In addition, it has been shown that changes in post-injection timeout did not affect measures of peak reinforcing strength, suggesting that the primary dependent variable is not influenced by pharmacokinetic factors (e.g., cumulative dosing; see Martelle et al. 2008), which will be important when comparing $\mathrm{S}^{\mathrm{D}}$ and $\mathrm{S}^{\mathrm{R}}$ effects.

\section{Materials and methods}

Subjects Four experimentally naïve, adult male rhesus monkeys (Macaca mulatta) served as subjects. Body weights were determined monthly, and each monkey was fed enough food (LabDiet Monkey Chow, and fresh fruit and vegetables), in addition to banana-flavored food pellets (Bio-Serv, Frenchtown, NJ, USA) earned during experimental sessions, to maintain weights at approximately $95 \%$ of free-feeding weight. Water was continuously available via drinking spouts installed in each cubicle. All experimental protocols and environmental enrichment were approved by the Institutional Animal Care and Use Committee.

Apparatus Monkeys were individually housed in ventilated, sound-attenuating cubicles $(91 \mathrm{~cm} \mathrm{~h} \times 91 \mathrm{~cm} \mathrm{w} \times 91 \mathrm{~cm}$ d, Plas Labs, Lansing, MI, USA) that also served as experimental chambers. Each monkey was fitted with a stainless steel restraint harness and spring arm (Restorations Unlimited, Chicago, IL, USA) that attached to the rear of the cubicle. The front panel of each chamber was clear Plexiglas to allow the monkey visual access to the laboratory and other conspecifics. Two metal boxes $(12.5 \mathrm{~cm} \times 15 \mathrm{~cm})$, each containing one response lever 
(PRL-001，BRS/LVE, Beltsville, MD, USA) and four stimulus lights, alternating red and white, were mounted $23 \mathrm{~cm}$ apart on the Plexiglas door. Additionally, a photo optic ergonomic switch (Stewart Ergonomics, Furlong, PA, USA) was mounted centrally on the Plexiglas door, and an orange stimulus light was located directly above the photocell. A food-pellet dispenser (G5210, Model A, Gerbrands, Arlington, MA, USA) was attached to the outside top of the Plexiglas door and connected to the food receptacle located between the two response levers by a length of Tygon tubing. An infusion pump (Cole-Parmer, Chicago, IL, USA) was located on the top of the chamber for delivery of IV drug solution during experimental sessions at a rate of $\sim 1.5 \mathrm{ml} / 10 \mathrm{~s}$. Computer and software controlled all experimental events and recorded data. Lighting was maintained on a 14-h-on, 10-h-off schedule occurring at 0600:2000 hours.

Catheter implantation Each monkey was prepared with a chronic indwelling venous catheter and subcutaneous vascular access port (Access Technologies, Skokie, IL, USA) under sterile surgical conditions using ketamine (15 mg/kg, i.m.) and butorphanol (0.05 mg/kg, i.m.) anesthesia. An incision was made near a major vein (e.g., femoral or jugular), and the catheter was inserted into the vein and advanced a distance calculated to terminate at the level of the vena cava. The distal end of the catheter was tunneled subcutaneously to a point on the back just lateral to midline at the level of the shoulder blades where an incision was made for the insertion of the vascular access port. The distal tip of the catheter was connected to the access port; the port was secured within the subcutaneous pocket that had been formed by blunt dissection, and the incisions were closed with sutures. Following closure, the area of skin above the vascular access port was cleaned with Betadine and 95\% ethanol, and a 22-gauge Huber Point Needle (Model PG20-125, Access Technologies) was inserted into the port. The animal was returned to its home cubicle where the external catheter tubing was threaded through the spring arm to the outside of the chamber and connected to the infusion pump. To maintain patency of the port and catheter, the system was filled with heparinized saline $(100 \mathrm{U} / \mathrm{ml})$ at the end of each experimental session.

\section{Experimental procedure}

Lever training Initially, each monkey was trained to lever press under an FR 1 schedule of food presentation. One lever was active, and the white stimulus lights above that lever were illuminated. The sequence of lever activity (right, R; left, L) was quasi-random across days and occurred as follows: RRLLRLLRRL. Over approximately 2 weeks, the ratio value was increased to 50. Completion of an entire ratio on the active lever resulted in food presentation. During pellet delivery, the white stimulus lights extinguished and the red stimulus lights were illuminated for $2 \mathrm{~s}$ followed by a $10-\mathrm{s}$ time-out (TO) in which responses emitted on the levers had no programmed consequence. Following the 10-s TO, the white stimulus lights illuminated, signifying the availability of another FR 50 and food presentation. These training sessions lasted for $60 \mathrm{~min}$ or until 30 food pellets had been earned. Once responding was reliably maintained by food presentation $( \pm 15 \%$ of the mean of three consecutive sessions with no trend), the monkey was prepared with catheter and port as described above.

Cocaine SA under a PR reinforcement schedule Monkeys were trained to discriminate self-administered cocaine from saline during morning sessions (0800); the cocaine or saline stimulus was delivered contingent on either right or left lever responding (randomly determined each day), and to selfadminister that substance (on the same lever) under a PR reinforcement schedule in evening sessions beginning at 1400 hours. The first injection was available under an FR 50 schedule; subsequent injections were available with increases in response requirement as determined by the exponential equation used by Richardson and Roberts (1996): ratio= $\left[5 \mathrm{e}^{(\text {injection } \# \times 0.2)}\right]-5$. For these experimental sessions, the first ratio requirement corresponded with the 12th value given by this formula (i.e., 50 responses). Completion of a ratio resulted in activation of the infusion pump and drug delivery for $10 \mathrm{~s}$. During the infusion, the stimulus lights changed from white to red. Following the infusion, there was a 15-min TO in order to minimize additive drug plasma concentrations. All stimulus lights extinguished for the duration of the TO, and responses had no programmed consequence. Once the TO expired, the white stimulus lights illuminated signaling the availability of another ratio and drug presentation. Sessions lasted until the animal failed to complete a ratio and receive an injection within 2-h (i.e., limited hold). The last ratio completed was defined as the breakpoint. Thirty minutes after the end of the session, the computer automatically triggered the infusion pump to deliver heparinzed saline for $30 \mathrm{~s}$ in order to flush the catheter of any residual drug. The sequence of cocaine (C) or saline (S) presentation matched the presentation during discrimination training. Monkeys had access to PR SA for 30 sessions during which baseline (saline and $0.15 \mathrm{mg} / \mathrm{kg}$ cocaine) breakpoints were established. To minimize cocaine exposure, the afternoon SA sessions were discontinued after determination of the baseline breakpoints.

Training self-administered cocaine as a discriminative stimulus Following catheter implantation, each monkey progressed to a morning DD training session beginning at 
0800 hours. Prior to the start of the session, the infusion pump was activated for approximately $15 \mathrm{~s}$ to fill the port and catheter with saline or the dose of cocaine available for SA. During DD training, monkeys responded under a twolink chained schedule of reinforcement. In the first link of the chained schedule, only one lever was active, and the white stimulus lights above that lever were illuminated. Monkeys responded under an FR 50 schedule to administer a single injection of cocaine $(0.15 \mathrm{mg} / \mathrm{kg})$ or saline. During the 10-s infusion, the stimulus lights changed from white to red. Following the injection, there was a TO (15 min for R1523 and R-1524; 20 min for R-1525 and R-1526, individually determined to optimize discrimination performance) prior to the start of the DD component. For the first 8 (R-1524) or 16 (R-1523, R-1525, R-1526) sessions, the presentation of $\mathrm{C}$ or $\mathrm{S}$ alternated every fourth day beginning with 4 days of cocaine presentation. Then, the sequence of $\mathrm{C}$ or $\mathrm{S}$ presentation was quasi-random across days and occurred as follows: SSCCSCCSSC. The active lever in this link also varied randomly and was not associated with cocaine- or saline-appropriate levers in DD. Following the TO, both levers were active, and the white stimulus lights above each lever illuminated signifying the beginning of the second link. In the second link of the chained schedule, monkeys discriminated the self-administered dose by responding under an FR schedule (FR 50 for R-1523, R1524, and R-1525; FR 30 for R-1526) of food presentation. Prior to the beginning of this experimental series, lever assignments were designated for each animal. For two monkeys, the right lever represented saline-appropriate, and the left lever represented cocaine-appropriate responding, while the lever assignments were reversed for the other two monkeys. During this link, 30 or 50 consecutive responses on the injection-appropriate lever resulted in food presentation; incorrect lever responses reset the FR value on the correct lever. Delivery of the pellet was accompanied by stimulus light change from white to red and then a 10-s TO with all lights extinguished. After the 10-s TO, the white lights above both levers illuminated, signifying the availability of another FR and food presentation. Conversely, 30 or 50 consecutive responses emitted on the inappropriate lever resulted in the same stimulus light change and 10-s TO but no food presentation. This link lasted for $15 \mathrm{~min}$ or until the animal received 50 pellets. Consistent cocaine discrimination was not achieved within 200 sessions; therefore, in an effort to obtain greater stimulus control of responding, a third operandum was installed in the experimental chamber, and the training dose was increased $(0.45 \mathrm{mg} / \mathrm{kg}$ for R-1523, R-1525, and R-1526; $0.56 \mathrm{mg} / \mathrm{kg}$ for R-1524). All drug self-administered during link 1 was now contingent upon finger-pokes emitted on a photooptic switch, signaled by illumination of an orange stimulus light directly above the switch, while food presentation during link 2 remained contingent upon lever pressing, signaled by illumination of white lights above each lever. Once discrimination responding was stable $(\geq 80 \%$ of responses prior to the first reinforcer and $\geq 90 \%$ of the total responses on the injection-appropriate lever), test sessions occurred.

Cocaine discrimination testing Test sessions were identical to training sessions with the exception that completion of the ratio requirement on either lever was reinforced. During test sessions, varying doses of cocaine (saline, 0.015$0.56 \mathrm{mg} / \mathrm{kg}$ ) were made available in the first link of the chained schedule, which served as the discriminative stimulus for the second link (DD). Presentation of drug doses during test sessions occurred in random order, and all drug doses were presented at least twice to each animal. Test sessions did not occur more than twice per week and not unless responding was deemed stable in four out of the five previous training sessions.

Combined DD and SA testing Once complete DD doseresponse curves were determined, monkeys were given access to the multiple two-link chained DD and PR SA schedule of reinforcement in the same session (Fig. 1). Sessions began with illumination of the orange stimulus

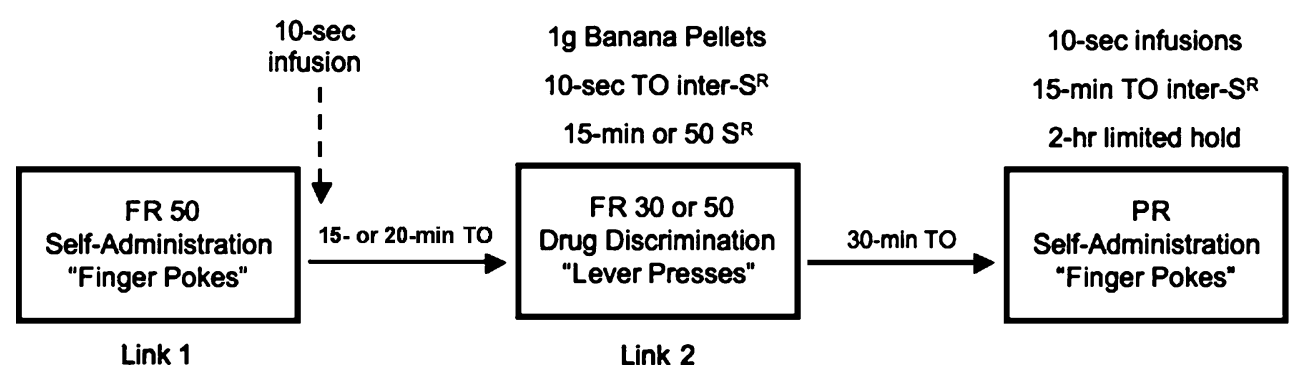

Fig. 1 Schematic of final parameters for multiple chained schedule of cocaine discrimination and self-administration. Link 1 consisted of an FR 50 schedule of self-administration that terminated in a single 10 -s injection followed by a TO. The injection from Link 1 served as the discriminative stimulus for Link 2 which was a two-lever, food-

reinforced drug discrimination task that lasted until 50 reinforcers were delivered or for $15 \mathrm{~min}$ and was followed by a 30 -min TO. The second component of the multiple schedule consisted of self-administration under a PR schedule of reinforcement. Each injection was followed by a 15 -min TO 
light above the finger-poke apparatus and a single injection of saline or cocaine was available under an FR 50 schedule of reinforcement. Following the injection, there was a 15or 20-min TO (selected to optimize individual performance), and the second link began by illumination of the white lever lights above both levers. There was a 30-min TO following DD during which all stimulus lights were extinguished, and responses were recorded but had no consequence. Following the TO, the orange stimulus light above the finger-poke apparatus was illuminated signaling the availability of drug SA under a PR schedule as described above. When both DD and PR performance were stable (for PR, $\pm 20 \%$ of the mean number of injections for the previous three similar drug conditions with no trend), varying doses of cocaine (saline, $0.015-0.56 \mathrm{mg} / \mathrm{kg}$ ) were substituted for the saline or cocaine training dose according to the same rules governing determination of the DD doseresponse curve. Presentation of drug doses during test sessions occurred in random order, and all drug doses were presented at least twice to each animal.

Data analysis

Link 1: FR Self-Administration For all monkeys, the chained schedule began with an FR 50 schedule of cocaine or saline presentation. The primary dependent variable was response rate, calculated as 50 responses/time. For the training conditions (cocaine and saline), the last three sessions prior to the first test session were used as "baseline". Data are shown for each monkey as the mean $( \pm \mathrm{SD})$ of the last three determinations of each training condition.

Link 2: Drug discrimination The primary dependent variables were the percent of responses that occurred on the cocaine-associated lever and the response rate (total number of responses/length of the response period). Dose-response curves determined for individual monkeys are represented as the mean $( \pm \mathrm{SD})$ of all determinations for that animal. Drug doses that engendered at least $80 \%$ cocaine-appropriate responding were considered to have substituted for the discriminative stimulus effects of cocaine. For doses that reduced the rate of responding to levels where no reinforcers were obtained, the rate of responding was calculated and included; however, the percent cocaine-appropriate responding was not included in any analysis.

PR Self-administration The primary dependent variables were the number of injections received and the final ratio value (i.e., breakpoint) completed before expiration of the limited hold. Data are represented as the mean $( \pm \mathrm{SD})$ number of injections and breakpoint for each monkey and as the mean $( \pm$ SEM) number of injections for the group. Under PR acquisition conditions, cocaine and saline data were analyzed using one-way and two-way repeated measures analysis of variance (ANOVA) tests followed by post hoc analyses using Dunnett's and Bonferroni's multiple comparison tests to assess whether responding maintained by cocaine was significantly higher than responding maintained by saline. Acquisition data were also analyzed using paired $t$ tests. Data from individual SA dose-response curve determinations were analyzed using one-way ANOVAs followed by Bonferroni's multiple comparison tests. All data were analyzed using Graphpad Prism (Graphpad Software, San Diego, CA, USA), and a $95 \%$ level of confidence $(p<0.05)$ was considered statistically significant.

Drugs (-)Cocaine $\mathrm{HCl}$ (National Institute on Drug Abuse, Bethesda, MD) was dissolved in $0.9 \%$ saline and diluted to concentrations of $0.3-5.0 \mathrm{mg} / \mathrm{ml}$. Cocaine was administered intravenously over 10 -s at a rate of approximately $1.5 \mathrm{ml} / 10 \mathrm{~s}$. Different doses of cocaine were studied by changing the concentration of drug available.

\section{Results}

Acquisition of PR cocaine SA Each day, monkeys had access to cocaine or saline twice, once in the morning where a single injection was available under an FR 50 schedule and the same dose (saline, $0.1 \mathrm{mg} / \mathrm{kg}$ cocaine) again in the afternoon under a PR schedule of reinforcement. Over the course of training, there were no differences in FR 50 response rates when saline or cocaine were available, with mean rates of responding of $1.56 \pm 0.47$ and $2.12 \pm 1.06$, respectively. The number of responses emitted and the number of injections obtained during the first 10 days of access to cocaine and 10 days of access to saline under the afternoon PR schedule of SA were analyzed as group data (Fig. 2a). Separate one-way ANOVAs revealed a significant effect of PR session number (i.e., experience with the PR schedule) under both cocaine and saline availability $\left(F_{3,9}=6.67, p<0.0001\right.$ and $F_{3,9}=3.10, p=$ 0.011 , respectively). A two-way ANOVA revealed significant main effects of experience and drug (i.e., cocaine or saline) as well as a significant interaction between the two $\left(F_{18,9}=5.72, p<0.0001\right)$. Post hoc analyses revealed that the cocaine and saline breakpoints significantly diverged by the third day of access to either condition (the seventh day of exposure to the PR schedule) and remained significantly different throughout the remaining 14 PR sessions analyzed. The mean $( \pm \mathrm{SD})$ number of injections and breakpoint for each monkey for the last five PR sessions of both 
Fig. 2 Acquisition of cocaine self-administration under a progressive-ratio schedule of reinforcement. a Group $(n=4)$ mean $( \pm$ SEM) number of injections self-administered during the first 20 sessions under a PR schedule of reinforcement. b Individual mean $( \pm \mathrm{SD})$ number of injections and breakpoints for the last 5 days of a 30-day access period to PR self-administration. Asterisks indicate significant $(p<0.05)$ difference from saline. During acquisition, the cocaine dose was $0.1 \mathrm{mg} / \mathrm{kg}$ per injection a.
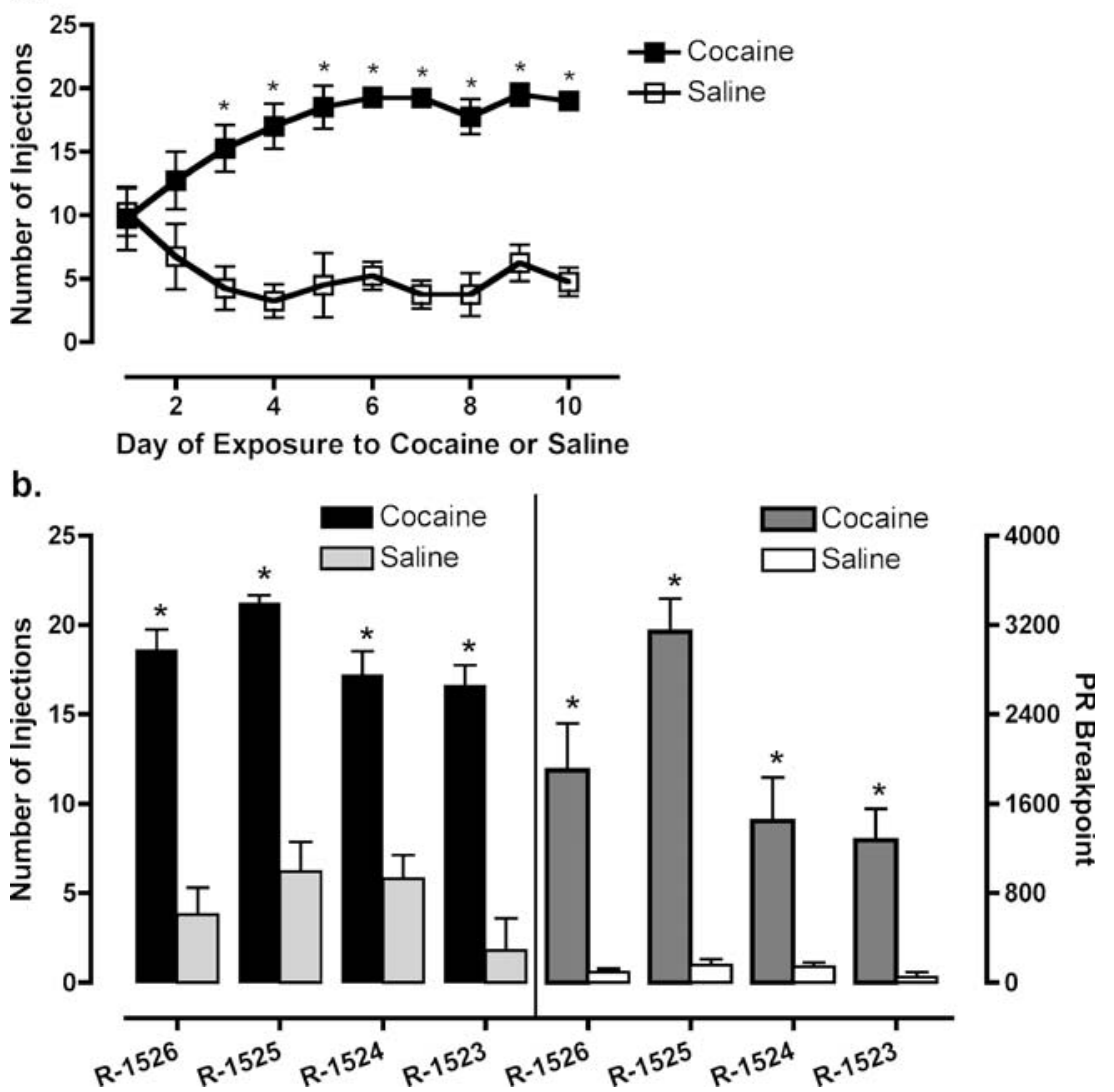

cocaine and saline presentation were compared to determine the stability of breakpoints under a quasi-random schedule of $\mathrm{C}$ vs. S presentation (Fig. 2b). Paired $t$ tests revealed that under these conditions the numbers of injections and breakpoints were significantly higher when cocaine was available compared to when saline was available. For each monkey under both the saline and cocaine condition, each of the breakpoints for the last five sessions was within $20 \%$ of the mean breakpoint for the previous three sessions of that condition. There were no differences in breakpoints observed under PR SA contingent upon lever presses vs. finger pokes.

Cocaine discrimination The number of training sessions necessary to obtain stable and reliable cocaine discrimination ranged from 221 to 334 (data not shown). The first 200-220 sessions of training involved using levers for both the first link (FR 50 saline or cocaine) and second link (food-reinforced discrimination training) at an intermediate cocaine dose $(0.1 \mathrm{mg} / \mathrm{kg})$. When the cocaine dose was raised and an additional manipulandum was added to the chamber such that cocaine or saline was contingent on finger pokes, while the operant for discrimination remained lever pressing, stable discrimination performance was established within 35-107 sessions. Contingent saline presentation occasioned $0 \%$ cocaine-appropriate responding, while the training dose of cocaine occasioned $100 \%$ cocaine-lever responding. Response rates in the discrimination link on saline sessions ranged from 1.0 to 2.67 vs. 0.50 to 1.67 responses/s when cocaine was self-administered in the first link (Fig. 3; open squares). In all monkeys, selfadministered cocaine $(0.045-0.9 \mathrm{mg} / \mathrm{kg})$ dose-dependently increased the percent cocaine-appropriate responding with full substitution observed with the training dose and higher cocaine doses (Fig. 3, filled squares). As a group, response rates did not vary with cocaine dose except at the highest dose.

Combined cocaine discrimination and self-administration Cocaine $(0.045-0.56 \mathrm{mg} / \mathrm{kg})$ dose-dependently increased the number of injections self-administered under a PR schedule of reinforcement in all monkeys (Fig. 4, open squares). Individual one-way ANOVAs for each monkey revealed that breakpoints were significantly higher when cocaine $(0.045$ $0.56 \mathrm{mg} / \mathrm{kg}$ ) was available for self-administration compared to when saline was available $\left(F_{3,4}=9.27, p=0.016 ; F_{3,8}=\right.$ $12.49, p=0.002 ; F_{2,3}=43.68, p<0.001 ; F_{2,3}=81.49, p=$ $0.002)$. There were no doses of cocaine that substituted for the training dose of cocaine during DD that did not also serve as a reinforcer during PR SA. In contrast, in each 
Fig. 3 Discrimination of selfadministered cocaine. The effect of varying doses of cocaine on cocaine discrimination (closed squares) and response rates (open squares). Each point is the mean $( \pm \mathrm{SD})$ of at least two determinations in each monkey. Arrows below the abscissa indicate the training dose of cocaine

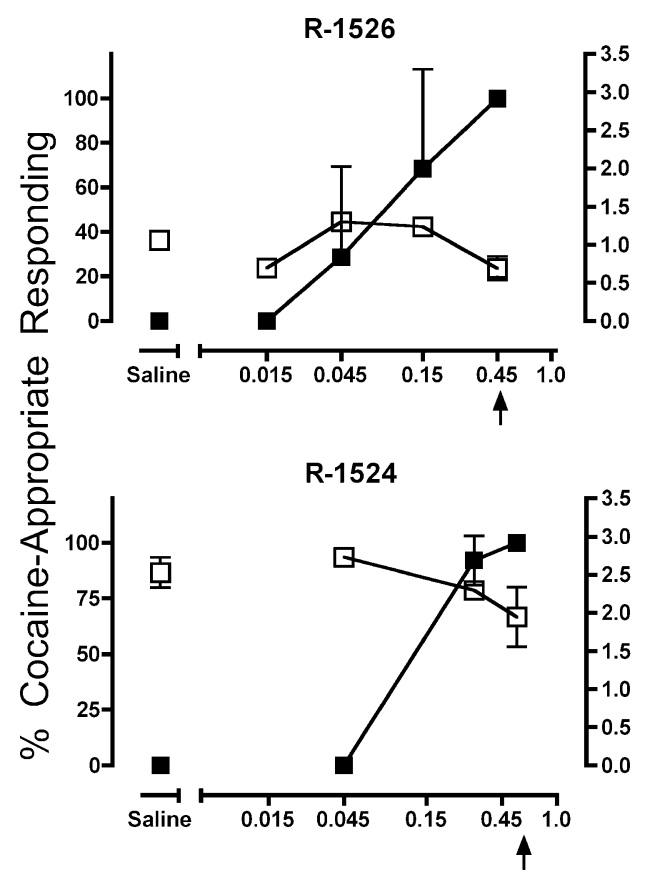

R-1525

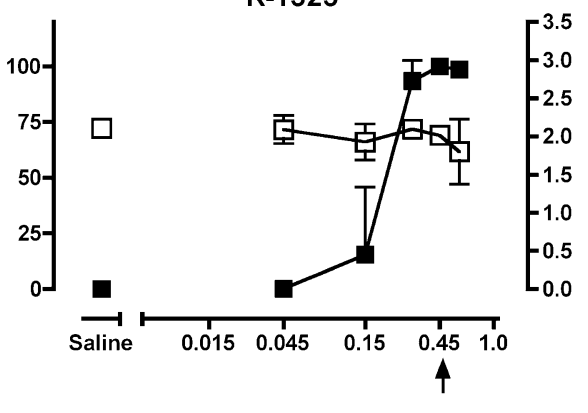

R-1523

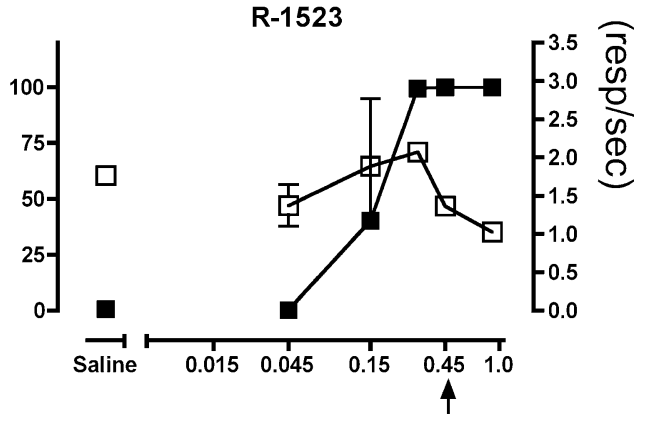

Cocaine Dose $(\mathrm{mg} / \mathrm{kg})$

monkey, there was at least one cocaine dose that failed to engender cocaine-appropriate responding during DD yet functioned as a reinforcer during SA (Fig. 4, open and closed squares). There was a significant positive correlation between the percent cocaine-appropriate responding engendered by a dose of cocaine and the number of injections selfadministered under the PR schedule of reinforcement $\left(r^{2}=\right.$ $0.59, p<0.01$; Fig. 5). When saline was available for self-

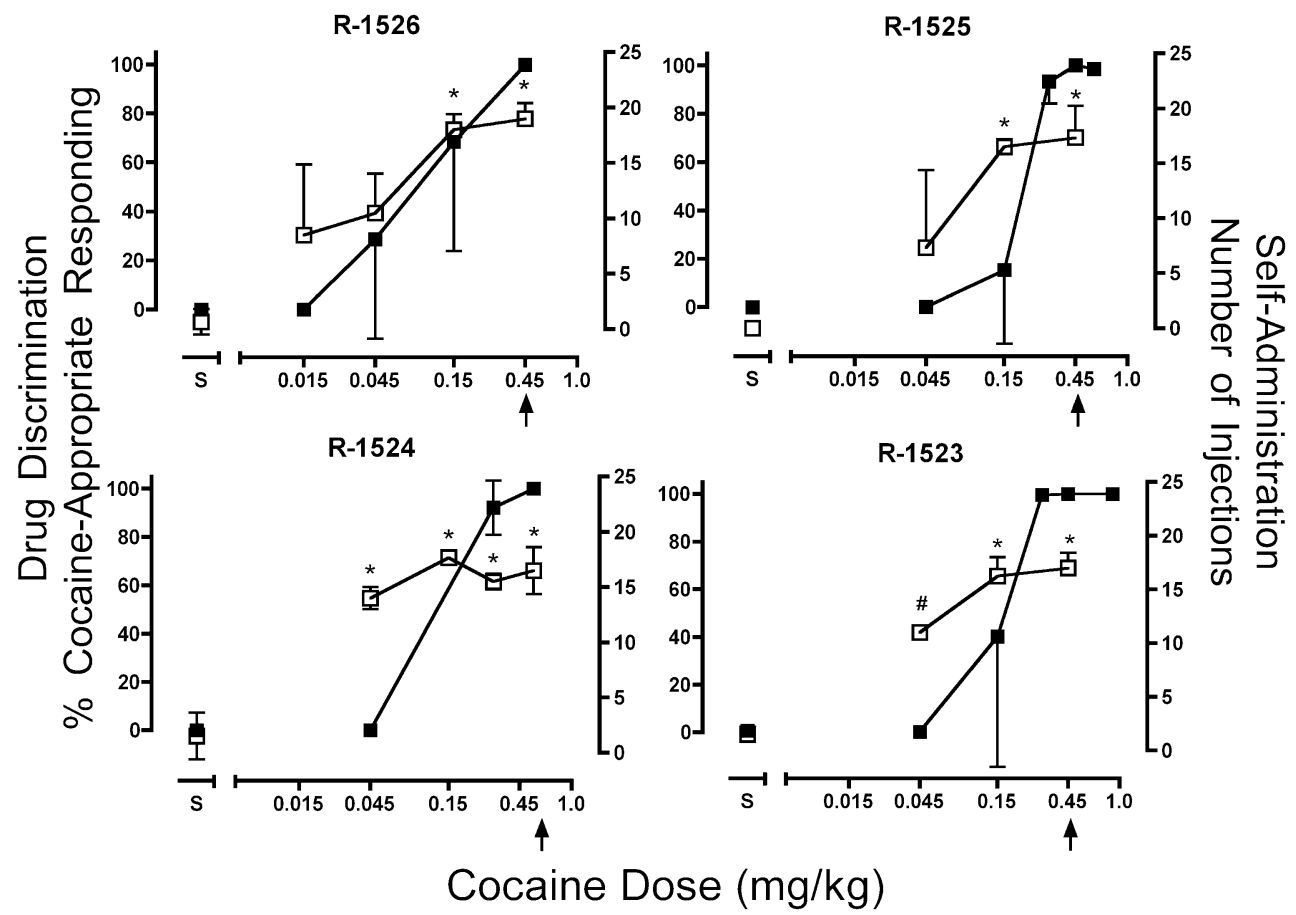

Fig. 4 The discriminative stimulus and reinforcing effects of selfadministered cocaine assessed under a multiple chained DD, PR SA schedule of reinforcement. The effect of various cocaine doses (abscissa; $\mathrm{S}$, saline) on the percent cocaine-appropriate responding (closed squares, left ordinate) during cocaine discrimination and number of injections (open squares, right ordinate) self-administered under a PR schedule of reinforcement. Each point is the mean $( \pm \mathrm{SD})$ of at lease two determinations in each monkey. Asterisks indicate significant $(p<0.05)$ difference from saline. Pound sign indicates single determination. Arrows below the abscissa indicate the training dose of cocaine used in DD link of the chained schedule of reinforcement. For R-1524, the $0.15 \mathrm{mg} / \mathrm{kg}$ cocaine dose was studied under the PR schedule during training, but not after DD performance had been established. All other points reflect data from the combined schedule 


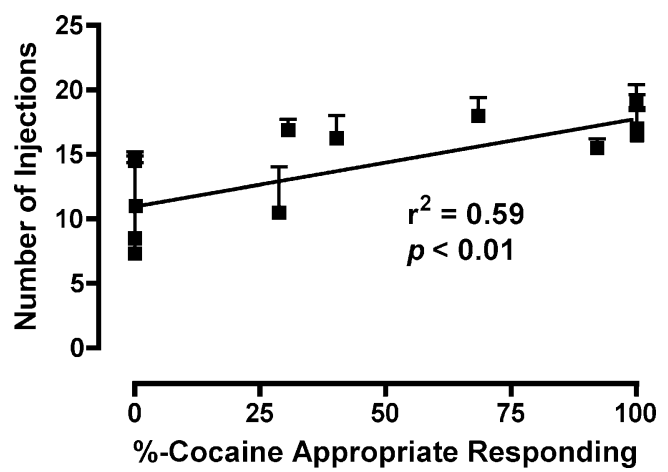

Fig. 5 Cocaine self-administration as a function of discriminative stimulus generalization. Individual mean $( \pm \mathrm{SD})$ number of injections (ordinate) of cocaine $(0.015-0.56 \mathrm{mg} / \mathrm{kg})$ self-administered under a PR schedule graphed as a function of the percent cocaine-appropriate responding (abscissa) engendered by those same doses of cocaine

administration following DD, monkeys frequently did not self-administer a single injection during the PR schedule (Table 1). This is in contrast to lower doses of cocaine that engendered saline-like responding during DD but were selfadministered at levels significantly higher than saline.

\section{Discussion}

The present study had two main objectives. First, a cocaine vs. saline discrimination was established in monkeys under conditions in which the training stimuli were self-administered. We found that in all monkeys, self-administered cocaine could be reliably discriminated from self-administered saline. Second, cocaine dose-response curves were generated under both DD and SA, within-subject, to determine whether sensitivity and efficacy to both stimulus effects were similar. In all monkeys following at least one cocaine dose, there was a dissociation between the cocaine-like $S^{D}$ effects and the $S^{R}$ effects of cocaine, such that a cocaine dose that occasioned saline-appropriate responding in DD, functioned as a reinforcer under the PR reinforcement schedule. These findings extend work conducted in humans and provide a novel model on which to evaluate potential cocaine treatments.

The main goal of the present study was to establish a novel behavioral model of drug abuse that combined DD and SA for the analysis of cocaine stimulus effects using a within-subjects design. The paradigm used in this study was similar to that used by Ator (2002) to study the effects of self-administered midazolam in baboons, although different training sequences and SA schedules of reinforcement were used. This is the first demonstration in animals that self-administered cocaine could be trained as a discriminative stimulus. The number of sessions required to establish the discrimination was substantially longer than reported by us or others using noncontingent cocaine (e.g. Nader et al. 1997; Sinnott et al. 1999), although the mechanism for such differences is not clear. One possibility involves the operandum used to deliver the training solutions. When SA and DD involved lever pressing, acquisition of the discrimination was slow. However, when we made the SA operandum distinct from the DD operandum, acquisition of cocaine discrimination appeared to be facilitated. Also of relevance for training in the present study was that the TO following cocaine selfadministration had to be lengthened from $10 \mathrm{~min}$, a time previously used by our laboratory (e.g., Nader et al. 1997) to $15-20 \mathrm{~min}$ in order to achieve stimulus control of behavior. In addition, the training dose of IV cocaine required for rhesus monkeys was higher in the present study ( 0.45 or $0.56 \mathrm{mg} / \mathrm{kg}$ ) compared to previous studies of noncontingent IV $(0.1 \mathrm{mg} / \mathrm{kg}$; Koetzner et al. 1996) and intramuscular (IM; 0.1-0.3 mg/kg; Nader et al. 1997; Sinnott et al. 1999; Martelle et al. 2007; $0.4 \mathrm{mg} / \mathrm{kg}$; Lamas et al. 1995, 1996; Negus et al. 2007) cocaine discrimination. Furthermore, the discrimination dose-response curves generated in the present study were shifted to the right of dose-response curves from previous studies (e.g., Koetzner et al. 1996; Nader et al. 1997), probably due to the higher training dose. Taken together, these results suggest that contingently administered cocaine can be trained as a $\mathrm{S}^{\mathrm{D}}$, although that effect may be less salient than noncontingently administered cocaine.

Table 1 Responses of a representative monkey during daily training and test sessions

Responding during combined drug discrimination and PR self-administration

\begin{tabular}{llrrrr}
\hline Subject & Dose (mg/kg) & Percent Coc appropriate responding & DD rate (resp/s) & PR responses (total) & No. of PR injections \\
\hline R-1525 & 0.45 Coc & 99.70 & 1.12 & 12164 & 0 \\
& Sal & 0.00 & 1.89 & 8683 & 0 \\
& 0.1 Coc & 0.23 & 1.89 & 9635 & 17 \\
& 0.45 Coc & 100.00 & 0.39 & 52 & 18 \\
\hline
\end{tabular}


With regard to training PR SA in previously cocainenaive monkeys, the cocaine SA dose-response curves generated in the present study were qualitatively (i.e., shape) and quantitatively (i.e., potency and efficacy) similar to those generated in previous studies from our laboratory using cocaine-experienced monkeys and in which each drug condition was available for multiple sessions until breakpoints were stable (e.g., Lile et al. 2002, 2003; Czoty et al. 2006). Most recently, we examined how TO duration would affect measures of reinforcement under a PR schedule identical to that used in this study (Martelle et al. 2008). This is particularly relevant because it could be argued that drug accumulation under the PR will lead to a larger "stimulus effect" compared to the single injection that occurred prior to DD. We found that TO manipulations only affected the low doses of cocaine $(0.015$ and $0.045 \mathrm{mg} / \mathrm{kg}$ ); the discrepancy between $\mathrm{S}^{\mathrm{D}}$ and $\mathrm{S}^{\mathrm{R}}$ was observed in the present study at a dose 1.0 log-units higher than cocaine doses affected by the TO. In addition, for most monkeys, when saline was the stimulus, they frequently self-administered less than five injections before the 2-h limited hold expired. Thus, it appears that the differences in $S^{D}$ and $S^{R}$ effects of cocaine are not due to drug accumulation under the SA schedule, although future studies using different reinforcement schedules are needed.

Lastly, but most importantly, the results from the combined DD and SA experiments emphasize the need for preclinical models that allow for the direct comparison of the $S^{D}$ and $S^{R}$ effects of drugs using a within-subjects design. Other studies in which nonhuman primates were trained to discriminate and self-administer either codeine (Hoffmeister 1988) or midazolam (Ator 2002) from drug vehicle have reported that these drugs were also more potent in the production of $S^{R}$ effects than in the production of $S^{D}$ effects necessary for DD. In each of these studies, there were examples of doses that did not engender drugappropriate responding during the DD task, yet functioned as a reinforcers during SA, which supports the conclusion that "the discriminative effect of a drug ... is not isomorphic with its reinforcing effectiveness" (Ator 2002 p. 477). Currently, preclinical studies of cocaine abuse use separate groups of animals to examine DD and SA despite the fact that human studies indicate baseline individual characteristics as a significant determinant of the subjective effects of drugs and that positive subjective effects are not tantamount with reinforcing effects (Evans and Griffiths 1992; de Wit et al. 1986a, b; Fischman 1989; Johanson and Uhlenhuth 1980, 1981, 1982). When evaluating potential pharmacotherapies, the possibility arises that important baseline individual variability may translate to differential response to therapy, which in turn, could inadequately be interpreted as equivocal therapeutic efficacy under a group design study. Moreover, drug treatments may differentially block one stimulus effect over another, and it may be shown, for example, that the best pharmacotherapy blocks the $S^{R}$ effects of cocaine, but not the $S^{D}$ effects. In both situations, the ability to assess a pharmacotherapeutic intervention under a within-subject design would be helpful.

In summary, DD and SA serve two important functions in searching for adequate pharmacotherapies to combat cocaine dependence. First, by attempting to attenuate the $\mathrm{S}^{\mathrm{D}}$ effects via agonist or antagonist treatment, DD paradigms offer excellent platforms for uncovering the CNS mechanism of drug action (Holtzman and Locke 1988). Second, by establishing that a treatment drug is able to decrease or attenuate cocaine self-administration, these paradigms are useful for evaluating potential pharmacotherapies (Mello and Negus 1996). Of course, there are caveats and potential limits to each approach, which are also evident in the present paradigm. For example, the impact of the cocaine training dose in drug discrimination can influence the sensitivity of drugs to substitute for cocaine (e.g., Terry et al. 1994 and Witkin et al., 1991), as is true with most drugs (e.g., Mumford and Holtzman 1991; Young et al. 1992). It is important to note that the training dose was the lowest dose that resulted in reliable discrimination under these conditions. A second potential limitation is the schedule of reinforcement used in the SA component. As mentioned above, it remains to be determined whether the dissociation observed between DD and SA was due to different stimulus effects or to the schedule of reinforcement used in the self-administration paradigm. Nonetheless, the novel model described in the present study provides an opportunity to directly compare the effects of potential treatments on the $\mathrm{S}^{\mathrm{D}}$ and $\mathrm{S}^{\mathrm{R}}$ effects of cocaine in the same subject during a single session; such a paradigm may be useful in the pursuit of effective pharmacotherapies.

Acknowledgements The authors are grateful for the technical assistance of Susan H. Nader and Tonya L. Calhoun and the advice of Nancy Ator and Paul Czoty. This research was supported by NIDA grant F30 DA021920 and P50 DA06634. These experiments represent a portion of the work conducted by JL Martelle as part of her dissertation research. The authors declare no financial conflicts of interest related to this research.

\section{References}

Ator NA (2002) Relation between discriminative and reinforcing effects of midazolam, pentobarbital, chlordiazepoxide, zolpidem, and imidazenil in baboons. Psychopharmacology 163:477-487

Bigelow GE, Preston KL (1989) Drug discrimination: methods for drug characterization and classification. NIDA Res Monogr 92:101-122

Brady JV, Lukas SE (1984) Testing drugs for physical dependence potential and abuse liability. NIDA Res Monogr 52

Carroll ME (1990) PCP and hallucinogens. Adv Alcohol Subst Abuse 9:167-190 
Colpaert FC (1999) Drug discrimination in neurobiology. Pharmacol Biochem Behav 64:337-345

Czoty PW, Martelle JL, Nader MA (2006) Influence of abstinence and conditions of cocaine access on the reinforcing strength of cocaine in nonhuman primates. Drug Alcohol Depend 85:213220

de Wit H, Uhlenhuth EH, Johanson CE (1986a) Individual differences in the reinforcing and subjective effects of amphetamine and diazepam. Drug Alcohol Depend 16:341-360

de Wit H, Uhlenhuth EH, Hedeker D, McCracken SG, Johanson CE (1986b) Lack of preference for diazepam in anxious volunteers. Arch Gen Psychiatry 43:533-541

Dykstra LA, Preston KL, Bigelow GE (1997) Discriminative stimulus and subjective effects of opioids with mu and kappa activity: data from laboratory animals and human subjects. Psychopharmacology 130:14-27

Evans SM, Griffiths RR (1992) Caffeine tolerance and choice in humans. Psychopharmacology 108:51-59

Fischman MW (1988) Behavioral pharmacology of cocaine. J Clin Psychiatry 49(Suppl):7-10

Fischman MW (1989) Relationship between self-reported drug effects and their reinforcing effects: studies with stimulant drugs. NIDA Res Monogr 92:211-230

Fischman MW, Foltin RW (1991) Utility of subjective-effects measurements in assessing abuse liability of drugs in humans. Br J Addict 86:1563-1570

Grant KA (1999) Strategies for understanding the pharmacological effects of ethanol with drug discrimination procedures. Pharmacol Biochem Behav 64:261-267

Griffiths RR, Bigelow GE, Henningfield JE (1980) Similarities in animal and human drug-taking behavior. In: Mello NK (ed) Advances in substance abuse. JAI, Greenwich, CT, pp 1-90

Griffiths RR, Lamb RJ, Ator NA, Roache JD, Brady JV (1985) Relative abuse liability of triazolam: experimental assessment in animals and humans. Neurosci Biobehav Rev 9:133-151

Hoffmeister F (1988) A comparison of the stimulus effects of codeine in rhesus monkeys under the contingencies of a two lever discrimination task and a cross self-administration paradigm: tests of generalization to pentazocine, buprenorphine, tilidine, and different doses of codeine. Psychopharmacology 94:315-320

Holtzman SG (1985) Drug discrimination studies. Drug Alcohol Depend 14:263-282

Holtzman SG (1990) Discriminative stimulus effects of drugs: relationship to potential for abuse. In: Adler MW, Cowan A (eds) Modern methods in pharmacology: testing and evaluation of drugs of abuse. Wiley-Liss, New York, NY, pp 193-210

Holtzman SG, Locke KW (1988) Neural mechanisms of drug stimuli: experimental approaches. Psychopharmacol Ser 4:138-153

Johanson CE, Fischman MW (1989) The pharmacology of cocaine related to its abuse. Pharmacol Rev 41:3-52

Johanson CE, Uhlenhuth EH (1980) Drug preference and mood in humans: d-amphetamine. Psychopharmacology 71:275-279

Johanson CE, Uhlenhuth EH (1981) Drug preference and mood in humans: repeated assessment of d-amphetamine. Pharmacol Biochem Behav 14:159-163

Johanson CE, Uhlenhuth EH (1982) Drug preferences in humans. Fed Proc 41:228-233

Kamien JB, Bickel WK, Hughes JR, Higgins ST, Smith BJ (1993) Drug discrimination by humans compared to nonhumans: current status and future directions. Psychopharmacology 111:259-270

Kleven MS, Anthony EW, Woolverton WL (1990) Pharmacological characterization of the discriminative stimulus effects of cocaine in rhesus monkeys. J Pharmacol Exp Ther 254:312-317

Koetzner L, Riley AL, Glowa JR (1996) Discriminative stimulus effects of dopaminergic agents in rhesus monkeys. Pharmacol Biochem Behav 54:517-523
Lamas X, Negus SS, Hall E, Mello NK (1995) Relationship between the discriminative stimulus effects and plasma concentrations of intramuscular cocaine in rhesus monkeys. Psychopharmacology 121:331-338

Lamas X, Negus SS, Nader MA, Mello NK (1996) Effects of the putative dopamine D3 receptor agonist 7-OH-DPAT in rhesus monkeys trained to discriminate cocaine from saline. Psychopharmacology 124:306-314

Lelas S, Spealman RD, Rowlett JK (2000) Using behavior to elucidate receptor mechanisms: a review of the discriminative stimulus effects of benzodiazepines. Exp Clin Psychopharmacol 8:294 311

Lile JA, Morgan D, Freeland CS, Sinnott RS, Davies HML, Nader MA (2000) Self-administration of two long-acting monoamine transport blockers in rhesus monkeys. Psychopharmacology 152:414-421

Lile JA, Morgan D, Birmingham AM, Wang Z, Woolverton WL, Davies HM, Nader MA (2002) The reinforcing efficacy of the dopamine reuptake inhibitor 2beta-propanoyl-3beta-(4-tolyl)tropane (PTT) as measured by a progressive-ratio schedule and a choice procedure in rhesus monkeys. J Pharmacol Exp Ther 303:640-648

Lile JA, Wang Z, Woolverton WL, France JE, Gregg TC, Davies HM, Nader MA (2003) The reinforcing efficacy of psychostimulants in rhesus monkeys: the role of pharmacokinetics and pharmacodynamics. J Pharmacol Exp Ther 307:356-366

Martelle JL, Claytor R, Ross JT, Reboussin BA, Newman AH, Nader MA (2007) Effects of two novel D3-selective compounds, NGB 2904 [N-(4-(4-(2,3-dichlorophenyl)piperazin-1-yl)butyl)-9Hfluorene-2-carboxami de] and CJB 090 [N-(4-(4-(2,3-dichlorophenyl)piperazin-1-yl)butyl)-4-(pyridin-2-yl)benzami de], on the reinforcing and discriminative stimulus effects of cocaine in rhesus monkeys. J Pharmacol Exp Ther 321:573-582

Martelle JL, Czoty PW, Nader MA (2008) Effect of time-out duration on the reinforcing strength of cocaine assessed under a progressive-ratio schedule in rhesus monkeys. Behav Pharmacol, in press

Mello NK, Negus SS (1996) Preclinical evaluation of pharmacotherapies for treatment of cocaine and opioid abuse using drug selfadministration procedures. Neuropsychopharmacology 14:375424

Mumford GK, Holtzman SG (1991) Qualitative differences in the discriminative stimulus effects of low and high doses of caffeine in the rat. J Pharmacol Exp Ther 258:857-865

Nader MA, Grant KA, Davies HM, Mach RH, Childers SR (1997) The reinforcing and discriminative stimulus effects of the novel cocaine analog 2beta-propanoyl-3beta-(4-tolyl)-tropane in rhesus monkeys. J Pharmacol Exp Ther 280:541-550

Negus SS, Mello NK, Blough BE, Baumann MH, Rothman RB (2007) Monoamine releasers with varying selectivity for dopamine/ norepinephrine versus serotonin release as candidate "agonist" medications for cocaine dependence: studies in assays of cocaine discrimination and cocaine self-administration in rhesus monkeys. $\mathrm{J}$ Pharmacol Exp Ther 320:627-636

Richardson NR, Roberts DC (1996) Progressive ratio schedules in drug self-administration studies in rats: a method to evaluate reinforcing efficacy. J Neurosci Methods 66:1-11

Roberts DC (1993) Self-administration of GBR 12909 on a fixed ratio and progressive ratio schedule in rats. Psychopharmacology 111:202-206

Schuster CR, Balster RL (1977) The discriminative stimulus properties of drugs. In: Thompson T, Dews PB (eds) Advances in behavioral pharmacology. Academic, New York, pp 85-138

Schuster CR, Fischman MW, Johanson CE (1981) Internal stimulus control and subjective effects of drugs. NIDA Res Monogr $37: 116-129$ 
Sinnott RS, Mach RH, Nader MA (1999) Dopamine D2/D3 receptors modulate cocaine's reinforcing and discriminative stimulus effects in rhesus monkeys. Drug Alcohol Depend 54:97-110

Terry P, Witkin JM, Katz JL (1994) Pharmacological characterization of the novel discriminative stimulus effects of a low dose of cocaine. J Pharmacol Exp Ther 270:1041-1048

Uhlenhuth EH, Johanson CE, Kilgore K, Kobasa SC (1981) Drug preference and mood in humans: preference for $d$-amphetamine and subject characteristics. Psychopharmacol 74:191-194
Witkin JM, Nichols DE, Terry P, Katz JL (1991) Behavioral effects of selective dopaminergic compounds in rats discriminating cocaine injections. J Pharmacol Exp Ther 257:706-713

Woolverton WL, Nader MA (1990) Experimental evaluation of the reinforcing effects of drugs. In: Adler MW, Cowans A (eds) Modern methods in pharmacology: testing and evaluation of drugs of abuse. Wiley-Liss, New York, pp 165-192

Young AM, Masaki MA, Geula C (1992) Discriminative stimulus effects of morphine: effects of training dose on agonist and antagonist effects of mu opioids. J Pharmacol Exp Ther 261:246-257 